\title{
Producción científica en el estado de Chihuahua, 1999-2008: análisis de las publicaciones registradas por el ISI Web of Knowledge
}

\author{
Faustino Moreno-Ceja, \\ María del Rocío Zumaya-Leal * \\ José de Jesús Cortés-Vera **
}

Artículo recibido: 19 de noviembre de 2010.

Artículo aceptado: 10 de enero de 2012.

\section{RESUMEN}

Este trabajo de investigación se basa en las publicaciones producidas en una década por investigadores del estado de Chihuahua, México; el análisis es predominantemente cuantitativo y se llevó a cabo a través de técnicas bibliométricas teniendo como objetivo examinar la producción del conocimiento científico en el estado a partir de una revisión de los documentos incluidos en las bases de datos de la ISI Web of Knowledge durante 1999-2008. La metodología seguida se basó en la aplicación de indicadores bibliométricos tales como: producción, temática, tipología, idioma, colaboración, índice de coautoría, entre otros. En relación con los resultados se observó que la producción total reportada

Ambos autores pertenecen a la Universidad de Guadalajara. México. (Faustino: fmoreno@cucba.udg.mx); (María: rzumaya@cucba.udg.mx)

** Universidad Autónoma de Ciudad Juárez, México. jcortes@uacj.mx

INVESTIGACIÓN BIBLIOTECOLÓGICA, Vol. 25, Núm. 55, septiembre/diciembre, 2011, México, ISSN: 0187-358X. pp. 201-225 
para el periodo estudiado fue de 973 documentos; se encontró que tan solo 10 instituciones tienen presencia en el total de la producción, las temáticas principales de publicación fueron Ciencia de los materiales (28.98\%), seguida de Física $(26.10 \%)$; la tipología preferida fue el artículo (60.64\%), el idioma principal de publicación fue el inglés (93.53\%); en relación con el indicador de colaboración, la mayor parte fue internacional (39.47\%), donde el principal socio fue EUA $(52.08 \%)$; la media de autores por documento fue de 4.66.

Palabras clave: Producción científica; Bibliometría; Indicadores bibliométricos; Chihuahua.

\section{ABSTRACT}

Scientific production in the state of Chihuahua, 1999 2008: analysis of publications registered by ISI Web of Knowledge

Faustino Moreno-Ceja, María del Rocío Zumaya-Leal and José de Jesús Cortés-Vera

This paper is based on the publications produced in a decade by researchers in the State of Chihuahua, Mexico; the analysis is predominantly quantitative and was undertaken through bibliometric techniques, being its objective to examine the production of scientific knowledge in the state, considering as starting point the documents included in the database of ISI Web of Knowledge during 1999-2008. The methodology followed considered the application of bibliometric indicators such as: output, subject areas, typology, language, collaboration, number of authors contributing to each article, among others. Regarding output, it was observed that the total production reported for the period under study was of 973 documents; it was also found that only 10 institutions had a presence in the total outcome; the main subject areas were Materials science $(28.98 \%)$, followed by Physics $(26.10 \%)$; the preferred format was the article $(60.64 \%)$; the main language was English (93.53\%). Concerning collaboration: most of it was international $(39.47 \%)$, where the main partner was the United States $(52.08 \%)$; the rate of authors per document was 4.66.

Keywords: Scientific production; Bibliometrics; Bibliometric indicators; Chihuahua. 


\section{INTRODUCCIÓN}

— n la transición hacia las sociedades del conocimiento es necesario encon-

- $t$ trar información sobre indicadores cada vez más precisos que muestren la forma y el ritmo en que se avanza en esta evolución. Estos indicadores deben ser cada vez más específicos e ir más allá de proporcionar datos sobre lo que sucede en un nivel nacional, y proporcionar información sobre lo que sucede en las entidades y regiones, pues regularmente existen fuertes desigualdades en este aspecto dentro de un país, como sucede en el caso de México.

El trabajo parte de la idea de que para determinar el avance de un país o de una entidad federativa hacia las sociedades del conocimiento es importante conocer cuál es la infraestructura de que disponen para producir conocimiento científico, cómo funciona ésta y qué productos genera. Esto se complementa con factores sobre los que no se abunda en este estudio, como las facilidades para que los ciudadanos tengan acceso a la información generada localmente y más allá de las fronteras, y para que desarrollen las competencias necesarias para aprovecharla, todo esto a través de políticas públicas pertinentes.

El estudio pone especial énfasis en los contenidos producidos a partir de un análisis predominantemente cuantitativo y a través de técnicas bibliométricas. Estudios con esta perspectiva son prácticamente inexistentes en el caso de México, al menos hasta donde ha sido posible determinarlo a través de una exploración bibliográfica realizada por los autores; los que se han hecho están relacionados con temáticas específicas o con instituciones, y no se han encontrado estudios como el que aquí se presenta, que describan la producción de un estado del país.

\subsection{Panorama de la investigación en México}

En el aspecto de inversión en investigación, el país ha estado realizando esfuerzos que aún no han rendido los frutos esperados, pero busca llegar a invertir al menos el 1.0 por ciento del Producto Interno Bruto (PIB) en ciencia y tecnología. Según datos del Consejo Nacional de Ciencia y Tecnología (CONACyT) en el 2005 esta inversión alcanzó el 0.80 por ciento (CONACyT, 2007).

Con respecto al tema de la centralización de recursos, el Gasto Federal en Ciencia y Tecnología (GFCyT), que comprende: a) la investigación y desarrollo experimental, b) la educación y enseñanza científica y técnica (formación de recursos humanos a nivel de posgrado) y c) los servicios científicos y tecnológicos, fue captado en un 86.1 por ciento, en lo que corresponde al sector educativo, por cinco instituciones: la Universidad Nacional Autónoma 
de México (UNAM), el Centro de Investigación y de Estudios Avanzados; el Instituto Politécnico Nacional; la Universidad Autónoma Metropolitana y El Colegio de México, todas ellas con sedes principales en la Ciudad de México, (CONACyT, 2007). Las instituciones mencionadas publican cerca del 40 por ciento de las 109 Revistas Mexicanas de Investigación Científica y Tecnológica reconocidas por el CONACyT como de calidad (CONACYT, 2009).

En otro dato, de un total de 14,576 miembros del Sistema Nacional de Investigadores (SNI) registrados en el 2007, un 22.23 por ciento estaban inscritos a la UNAM, institución que además reúne el mayor porcentaje de investigadores de Nivel III, que es el máximo nivel otorgado; la Universidad Autónoma Metropolitana contaba con el 5.46 por ciento y el Instituto Politécnico Nacional el 4.39 por ciento. Al mismo tiempo, las dos universidades públicas del estado de Chihuahua, la Universidad Autónoma de Ciudad Juárez y la Universidad Autónoma de Chihuahua tenían cada una 48 investigadores en este registro, con lo que colaboraban las dos sumadas con un 0.66 por ciento al total nacional (Ordorika, et al, 2009).

Para tener una idea de la producción científica nacional con respecto a la del resto del mundo, puede mencionarse que México aportó, entre el 2002 y el 2006, el 0.75 por ciento de los artículos captados por el ISI Web of Knowledge, muy lejos del 32.97 por ciento que alcanzaron los Estados Unidos o del 3.25 logrado por España; distante todavía del 1.92 por ciento alcanzado por Brasil, aunque arriba del 0.58 por ciento logrado por Argentina y el 0.32 por ciento de Chile (CONACyT, 2007). Las principales áreas temáticas en las que se publica en el país son: física, química, plantas y animales, y medicina, que en conjunto sumaron más del 54 por ciento de la producción durante el mismo periodo (CONACyT, 2007).

\subsection{Infraestructura para la investigación en Chibuahua}

Buena parte de la producción científica que se realiza en México está a cargo de las instituciones de educación superior, principalmente de aquellas que cuentan con programas de doctorado. En el estado de Chihuahua, solamente las siguientes 5 instituciones cuentan con este nivel, entre un total de 614 similares identificadas en 2006 en todo el país, (CONACyT, 2007). El Centro de Investigación en Materiales Avanzados (CIMAV). El Instituto Tecnológico de Chihuahua (ITECH). El Instituto Tecnológico de Ciudad Juárez (ITCJ). La Universidad Autónoma de Chihuahua (UACH) y la Universidad Autónoma de Ciudad Juárez (UACJ).

Otra parte importante de la generación de conocimiento en México se realiza a través del sistema de los Centros Públicos de Investigación del CONACyT, 
del cual el CIMAV forma parte y es el único de los 27 centros existentes en el 2007 que tiene su sede principal en el estado de Chihuahua. Este sistema constituye una estrategia muy importante en la descentralización de las actividades científicas y tecnológicas de México; los centros adscritos contribuyen a conocer la realidad y los problemas de las diferentes regiones del país, desde diversos campos del conocimiento (CONACyT, 2008b).

Existen también otras instituciones del sistema de Centros Públicos de Investigación del CONACyT que tienen su sede principal en alguna otra parte del país, pero que han establecido una subsede en el estado de Chihuahua. Es el caso del Centro de Investigación en Alimentación y Desarrollo (CIAD), El Colegio de la Frontera Norte (COLEF) y el Instituto Nacional de Ecología (INE).

En cuanto a recursos humanos calificados para la investigación, en el 2007 el estado contaba con 153 investigadores miembros del SNI, de los cuales una tercera parte tenía el nivel de candidato y solamente un investigador había alcanzado el nivel III. Más de una tercera parte (55) de estos investigadores estaban ubicados en el área disciplinar de las Ciencias de la Ingeniería, seguidos por Biotecnología y Ciencias Agropecuarias con 36 y Física, Matemáticas y Geociencia con 18, (CONACyT, 2008).

\subsection{Limitaciones de las bases de datos}

Las limitaciones de las bases de datos del ISI han sido suficientemente discutidas en la literatura. Autores como Taubes (1993), Adam (2002) y Gálvez y Moya (2006), entre otros, resaltan los errores del ISI derivados de la captura de la información, lo que genera inconsistencias en datos como los nombres de los autores, su adscripción y la dirección de sus instituciones. Cabe señalar que estos errores en ocasiones no son imputables al ISI y su sistema de captura, sino a la falta de orden y consistencia de quienes suministran los artículos.

Una de las mayores desventajas de estas bases de datos es que no incluyen libros, capítulos de libros, ni tesis, lo que deja fuera una parte importante de la producción científica; existe también un sesgo preferente a incluir documentos en el idioma inglés, señalado por Braun, Glänzel y Schubert (1985). Otra desventaja que se ha observado tiene que ver con la pobre cobertura de las Ciencias sociales y las Humanidades, sobre todo cuando se trata de trabajos que abordan aspectos regionales (Lascurain, 2001).

Entre las ventajas de estas bases de datos podemos mencionar su carácter internacional, su cobertura multidisciplinar, la actualización casi inmediata, la inclusión de las referencias en cada trabajo reportado y la disponibilidad de formato para su consulta, entre otras. 


\section{Objetivo}

El objetivo de esta investigación fue el de analizar la producción científica del estado de Chihuahua mediante la aplicación de los indicadores bibliométricos: producción científica, temática, tipología, idioma y colaboración entre otros.

\section{Metodología}

En este apartado se describe la metodología utilizada en el proceso de la investigación para alcanzar el análisis planteado. A partir del uso de las bases de datos de los productos del ISI se extrajeron los registros del periodo (19992008), para ser observadas y así seleccionar la información, con el objeto de generar resultados que expliquen el comportamiento de la producción científica del estado de Chihuahua.

Como herramientas informáticas se utilizó Microsoft Office Word versión 2007, Microsoft Office Excel 2007, el gestor bibliográfico Procite 5.0 para Windows y Bibliolink II.

La estrategia de búsqueda seguida para la obtención de los registros fue el campo $\mathrm{AD}=$ Chihuahua, y se encontraron 986 registros que cumplieron con el requisito de incluir la palabra "Chihuahua" en el campo Address (03) para la obtener la institución o instituciones participantes, lo cual fue realizado el 17 de marzo de 2009.

Una vez obtenidos los registros que de entrada fueron 986, lo siguiente fue la tarea de filtrar, ordenar, codificar, normalizar y estandarizar, hasta llegar a la cifra de 973 . Con estos registros se procedió al análisis, mediante la aplicación de los indicadores bibliométricos, considerando como tales

los datos numéricos extraídos de los documentos que publican los investigadores o de los que utilizan los usuarios y que permiten analizar distintas características de su actividad científica, vinculadas, tanto a su producción como al consumo de su información (Sanz Casado y Martín Moreno, 1997).

- Producción científica. En este indicador se contabilizó el número de documentos recogidos de las bases de datos del ISI, dentro de las cuales se obtuvo la evolución de ésta a lo largo de los diez años analizados.

- Temática. Ésta se obtuvo realizando una separación de las temáticas que contiene cada revista, la cual puede contener una o varias. Posteriormente se realizó el conteo y de esta manera se obtuvo el número 
de frecuencias de éstas, para calcular los valores porcentuales a partir del total de los documentos publicados.

- Tipología. Se determinó mediante el conteo de los tipos de documentos elegidos para presentar la producción científica. Con las cantidades de cada una de las tipologías se establecieron las frecuencias para calcular los valores porcentuales con respecto del total de los documentos publicados.

- Idioma. Con la clasificación del idioma otorgada a cada trabajo se obtuvo la distribución y frecuencias de éstos, con los cuales se calcularon los valores absolutos y porcentuales para obtener el indicador.

- Dispersión. Con este indicador se identificó cuáles son las revistas más utilizadas por los científicos para dar a conocer sus investigaciones. Después se elaboró una tabla con las frecuencias absolutas y acumuladas de los diferentes títulos de revistas, así como del número de artículos de cada título y los porcentajes que representan frente al total de artículos. Ello permitió conocer los títulos que conforman el núcleo de revistas más productivas y establecer la primera zona.

- Colaboración. Para calcular el índice de cooperación o de colaboración se contabiliza el número de instituciones firmantes que han intervenido en un trabajo. En nuestro caso la matriz está formada por las frecuencias que se derivan de los documentos firmados conjuntamente por autores de dos o más instituciones del estado de Chihuahua). La clasificación seguida para este trabajo quedó determinada en: internacional, nacional, local y sin colaboración.

- Porcentaje de trabajos en colaboración. Éste se realizó identificando y contabilizando las instituciones que firman los registros obtenidos de las bases de datos utilizadas, haciéndolo de acuerdo con la clasificación que señalamos en el párrafo anterior.

- El total de coautoría se obtuvo mediante la suma de autores firmantes de los trabajos reportados entre el número de trabajos. El índice de colaboración se calculó a través del número de direcciones de centros de trabajo que han intervenido en la elaboración de una investigación, y su valor se obtuvo haciendo una división del total de instituciones firmantes de los trabajos entre el total de trabajos reportados.

- Impacto. El cálculo se realizó identificando cada uno de los títulos de revista en la temática asignada en las bases de datos JCR/SCI y JCR/ SSCI, mismos que se ordenaron a partir del título de la revista con el Factor de Impacto más alto y divididos entre 4 para obtener los cuarteles; de esta manera, el valor de las revistas ubicadas en el primer cuartil estaban dentro de $1 \%$ al $25 \%$, las del segundo del $26 \%$ al 
$50 \%$, el tercero entre el $51 \%$ y el $75 \%$ y las del cuarto y último entre el $76 \%$ y el $100 \%$.

Finalmente es importante señalar desde el punto de vista metodológico, que los recuentos se pueden realizar de diferentes formas: por primer autor, fraccionado y total (Cronin y Overfelt, 1994; Lange, 2001 y Maltrás, 2003). Este último fue el aplicado en esta investigación, razón por la cual las cifras de la sumatoria y porcentajes son distintas en algunos indicadores.

\section{Resultados}

\subsection{Producción cientifica}

La producción de documentos en la década 1999-2008 en el estado de Chihuahua, captada por el ISI, ascendió a 973 trabajos, como se observa en la Tabla I. Esto significa un promedio de 97.3 documentos por año. El año de menor producción fue el primero, 1999, con solamente 28 trabajos, y el de mayor producción el 2007, con 165. Se observa una tendencia ascendente, interrumpida en el 2002, año en el que al parecer hubo problemas en todo el país, pues esta misma reducción se observó en el estudio realizado por Moreno (2010) para el caso de la Universidad de Guadalajara. En el contexto nacional no hubo una disminución, pero sí se registró un escaso crecimiento, pues apenas se pasó de 4,983 documentos producidos en el 2001 a 5,192 en el 2002 (CONACyT, 2007). También se observa una reducción de registros en el 2008, pero en este caso es una situación normal que se explica porque en el momento de la recolección de los datos aún no existían en las bases de datos todos los registros que aparecían con fecha de ese año.

Tabla I: Producción científica del estado de Chihuahua, 1999-2008

\begin{tabular}{ccccccccccc}
\hline 1999 & 2000 & 2001 & 2002 & 2003 & 2004 & 2005 & 2006 & 2007 & 2008 & Total \\
28 & 56 & 77 & 60 & 80 & 104 & 130 & 145 & 165 & 128 & 973 \\
\hline
\end{tabular}

Para darles contexto a estas cifras puede mencionarse que en la década comprendida entre 1997 y 2006, el país produjo en conjunto 52,029 artículos, (CONACyT, 2007). En los estados del país, Chihuahua ocupa el lugar 15 de los 30 estados reportados. Su producción es menor, por mencionar algunos ejemplos, a los 1,986 registros publicados en el mismo periodo de estudio, por el estado vecino de Sonora, a los 3,581 del estado de Jalisco, a los 2,008 de San Luis Potosí y a los 2,872 que en el mismo periodo fueron escritos por 
autores del estado de Guanajuato. Algunos estados como Baja California contaron con 3,822 registros; Morelos con 6,732 y Puebla con 5,123 que son cifras elevadas, en gran medida gracias a la producción realizada por centros nacionales de investigación o por filiales de instituciones con sede en la ciudad de México.

Cabe señalar que el Estado de México y el Distrito Federal no fueron incluidos, por dificultades para recuperar la información específica, en el caso del primero y porque el interés principal era comparar la información de los estados.

Estos datos son también concordantes con los publicados por Licea de Arenas, et al (2006) acerca de la investigación mexicana significativa en ciencias de la salud, pues los autores encontraron resultados muy similares, ya que los estados que reportan guardan el siguiente orden: Distrito Federal, Morelos, Querétaro, Puebla, Guanajuato, Nuevo León, Jalisco, etc.; estados que en nuestro estudio se encuentran de manera similar en los primeros lugares en relación con la cantidad de documentos producidos.

Como puede observarse en la Tabla II, el CIMAV fue responsable en este periodo de generar prácticamente la mitad de los documentos registrados por el ISI. Este centro y la UACH por sí solos produjeron tres de cada cuatro trabajos publicados. La tabla siguiente muestra las diez instituciones con mayor cantidad de producción.

Tabla II: Producción científica por instituciones del Estado de Chihuahua, 1999-2008

\begin{tabular}{|l|c|c|}
\hline \multicolumn{1}{|c|}{ Instituciones } & Frecuencias & Porcentaje \\
\hline CIMAV & 484 & 49,74 \\
\hline UACH & 266 & 27,34 \\
\hline UACJ & 83 & 8,53 \\
\hline ITECH & 63 & 6,47 \\
\hline IMSS-CH & 44 & 4,52 \\
\hline CIAD-CH & 25 & 2,57 \\
\hline INIFAP-CH & 17 & 1,75 \\
\hline ITECH 2 & 11 & 1,13 \\
\hline Hospital Clínica del Parque & 8 & 0,82 \\
\hline ITESM-CH & 7 & 0,72 \\
\hline Otras & 48 & 4,93 \\
\hline Sumatoria & 1056 & 108,53 \\
\hline Total de documentos & 973 & 100 \\
\hline
\end{tabular}




\subsection{Temática de la producción}

En la Tabla III pueden verse los temas principales cubiertos por el colectivo estudiado, siguiendo la clasificación utilizada por el ISI en sus 22 áreas temáticas. Para realizar el análisis de las materias se consideraron todos los temas incluidos en las revistas donde publicaron los investigadores del estudio en cuestión. Al respecto es importante señalar que el

ISI no clasifica los documentos de acuerdo con su contenido temático, sino que únicamente clasifica las revistas donde se han publicado estos trabajos. En función del producto que comercializa, el ISI utiliza distintas clasificaciones temáticas, que responden a diferentes agrupaciones de las revistas por disciplinas o áreas temáticas (Cami, et al., 2003).

Tabla III: Temática de la producción científica del estado de Chihuahua, 1999-2008

\begin{tabular}{|l|c|c|}
\hline \multicolumn{1}{|c|}{ Áreas temáticas (ISI) } & Frecuencias & Porcentaje \\
\hline Ciencia de los materiales & 282 & 28,98 \\
\hline Física & 254 & 26,10 \\
\hline Química & 207 & 21,27 \\
\hline Ingeniería & 204 & 20,97 \\
\hline Ciencias agrícolas & 126 & 12,95 \\
\hline Medicina Clínica & 114 & 11,72 \\
\hline Ecología y Medioambiente & 79 & 8,12 \\
\hline Biología y Bioquímica & 75 & 7,71 \\
\hline Informática & 69 & 7,09 \\
\hline Geociencias & 68 & 6,99 \\
\hline Ciencias Sociales & 57 & 5,86 \\
\hline Ciencias de las Plantas y Animales & 54 & 5,55 \\
\hline Microbiología & 26 & 2,67 \\
\hline Biología molecular y Genética & 25 & 2,57 \\
\hline Inmunología & 10 & 1,03 \\
\hline Matemáticas & 10 & 1,03 \\
\hline Farmacología & 9 & 0,92 \\
\hline Neurociencias y Comportamiento & 8 & 0,82 \\
\hline Psiquiatría y Psicología & 5 & 0,51 \\
\hline Ciencias del espacio & 1 & 0,10 \\
\hline Economía y Negocios & & 0,10 \\
\hline
\end{tabular}




\begin{tabular}{|l|c|c|}
\hline Ciencias multidisciplinares & 1 & 0,10 \\
\hline Sumatoria & 1685 & 173,16 \\
\hline Total de títulos & 973 & \\
\hline
\end{tabular}

Casi tres de cada diez artículos publicados tienen relación con las Ciencias de los materiales, la alta producción en esta temática es congruente con la presencia en el estado del CIMAV. La Física ocupa más de la cuarta parte de la producción con el 26.10 por ciento. El tercer tema en importancia es la Química, con un 21.27 por ciento, seguida muy de cerca por la Ingeniería, con casi un 21 por ciento. Como puede observarse existen diferencias en los temas más importantes abordados en el estado en comparación con el contexto nacional, en donde ya se mencionó que los temas principales fueron Física, Química, Plantas y animales, y la Medicina.

Los resultados concuerdan parcialmente con los obtenidos por Fernández, et al (2004) en su investigación relacionada con la especialización temática de los países de América Latina, donde las Ciencias de la Salud y la Física ocuparon los primeros lugares. De manera similar, Moreno (2010) encontró que en la Universidad de Guadalajara el primer lugar es para las Ciencias de la Salud y el segundo para la Física, seguidas por las Ciencias agrícolas y Ecología y Medio Ambiente, casi en el mismo orden también Fernández, Gómez y Sebastián (1998) reportaron Física, Biomedicina y la Medicina Clínica; seguidas del conjunto de materias formadas por Agricultura, Biología y el Medio Ambiente.

En este mismo sentido Ortiz Rivera (2002) en su trabajo sobre la actividad científica en Puerto Rico, destacó que el área de Ciencias de la vida y la Medicina en conjunto representan un 45,03\%, seguidos del área de Ciencia y tecnología con 30,38\% y la Agricultura y Medio ambiente, con un $13,11 \%$. Una tendencia parecida la siguen los países en vías de desarrollo identificada por Leta, Pereira y Chaimovich (2005) en su estudio del factor de impacto de las revistas donde publicaron los investigadores de la Universidad de São Paulo en Ciencias de la vida, ya que entre los países identificados como periféricos la mayor parte de sus publicaciones se da en el área de Ciencias de la Salud.

La producción de Ciencias Sociales aparece con cifras notoriamente bajas, a sabiendas de que es uno de los campos disciplinares en los que las dos universidades públicas tienen mayores fortalezas; esto es consecuencia de las ya mencionadas limitaciones que presentan las bases de datos del ISI para captar la producción científica en estos temas, sobre todo cuando incluyen coberturas y referentes regionales. 


\subsection{Tipología}

La Tabla IV muestra los resultados obtenidos con respecto al formato de la publicación. Dado que las bases de datos del ISI se nutren fundamentalmente del contenido de revistas, el artículo aparece como el principal formato utilizado.

Tabla IV: Tipología de la producción científica del estado de Chihuahua, 1999-2008

\begin{tabular}{|l|c|c|}
\hline \multicolumn{1}{|c|}{ Tipología } & Frecuencias & Porcentajes \\
\hline Artículos & 590 & 60,64 \\
\hline Actas de congresos & 249 & 25,59 \\
\hline Resumen de comunicaciones & 102 & 10,48 \\
\hline Revisiones & 14 & 1,44 \\
\hline Editoriales & 5 & 0,51 \\
\hline Cartas & 5 & 0,51 \\
\hline Revisiones de libros & 3 & 0,31 \\
\hline Noticias & 2 & 0,21 \\
\hline Biografías & 1 & 0,1 \\
\hline Correcciones & 1 & 0,1 \\
\hline Revisión de teatro & 1 & 0,1 \\
\hline Total & 973 & 100 \\
\hline
\end{tabular}

La inclusión en este estudio de las bases de datos Conference Proceedings Citation Index- Science (CPCI-S) y Conference Proceedings Citation Index- Social Science \& Humanities (CPCI-SSH) produce resultados que son diferentes a los de otros trabajos, como el presentado por Sancho et al (2006), quienes en un análisis de la colaboración científica en América Latina encontraron que el artículo representaba el 80 por ciento de la producción. Por su parte, Russell, Ainsworth y Narváez (2006), calculan que el 91 por ciento de los documentos producidos en la UNAM en el área de las ciencias básicas, tienen este formato.

\subsection{Idioma de la producción científica}

El 93.53 por ciento de los documentos analizados en este estudio fueron publicados en inglés, como puede observarse en la Tabla V. Esta cifra, a pesar de ser tan alta, debe considerarse como normal, en parte porque las bases de datos utilizadas para este estudio captan principalmente publicaciones periódicas escritas en este idioma, pero también porque, a final de cuentas, el inglés ha 
sido seleccionado por la mayor parte de la comunidad científica internacional para socializar sus conocimientos.

Tabla V: Idioma de la producción científica del estado de Chihuahua, 1999-2008

\begin{tabular}{|l|c|c|}
\hline \multicolumn{1}{|c|}{ Idioma } & Frecuencias & Porcentaje \\
\hline Inglés & 910 & 93,53 \\
\hline Español & 62 & 6,37 \\
\hline Alemán & 1 & 0,1 \\
\hline Total & 973 & 100 \\
\hline
\end{tabular}

Llama la atención que esta cifra coincide casi exactamente con la reportada por Russell, Ainsworth y Narváez (2006) para el estudio de la producción científica de la UNAM en el período de 1999 a 2002, en donde encontraron que el 93 por ciento de esa producción fue publicada en inglés, aunque un estudio posterior realizado por González, Gómez y Licea de Arenas (2008), elevaba este porcentaje a un 98 por ciento. Un resultado similar obtuvieron también Olmeda, et al (2008), en su estudio del impacto internacional de la producción científica de las universidades de Cataluña, con 94.58 por ciento de documentos en lengua inglesa.

El 6.37 por ciento de los documentos fue publicado en revistas que aceptan trabajos presentados en español y solamente un trabajo fue aportado en idioma alemán.

\subsection{Colaboración}

Los porcentajes reflejados en la Tabla VI muestran que este colectivo realiza en gran parte su trabajo en conjunto con investigadores de otras instituciones. Haciendo una suma de las colaboraciones, encontramos que el $72.76 \%$ se dieron en esta modalidad, en donde el $39.47 \%$ es de carácter internacional, mientras que la nacional obtiene un $29.29 \%$ y sólo $4.01 \%$ es entre instituciones del estado de Chihuahua; el restante $27.24 \%$ corresponde a los documentos sin colaboración.

Tabla VI. Comportamiento de la producción del estado de Chihuahua con respecto a la colaboración, 1999-2008

\begin{tabular}{|c|c|c|c|c|}
\hline Años & Internacional & Nacional & Local & Sin col. \\
\hline 1999 & 12 & 6 & 0 & 10 \\
\hline 2000 & 25 & 15 & 0 & 16 \\
\hline 2001 & 24 & 27 & 2 & 24 \\
\hline
\end{tabular}




\begin{tabular}{|l|c|c|c|c|}
\hline 2002 & 22 & 18 & 1 & 19 \\
\hline 2003 & 29 & 21 & 4 & 26 \\
\hline 2004 & 46 & 24 & 1 & 33 \\
\hline 2005 & 47 & 43 & 6 & 34 \\
\hline 2006 & 59 & 42 & 9 & 35 \\
\hline 2007 & 66 & 51 & 4 & 44 \\
\hline 2008 & 54 & 38 & 12 & 24 \\
\hline Totales & 384 & 285 & 39 & 265 \\
\hline Porcentajes & 39,47 & 29,29 & 4,01 & 27,24 \\
\hline
\end{tabular}

Si bien es cierto que la ubicación geográfica del estado le otorga algunas ventajas competitivas para emprender proyectos colaborativos internacionales, principalmente con los EU, también se contempla que existe un alto potencial de colaboración entre las instituciones chihuahuenses, sobre todo entre las dos instituciones con mayor producción en este trabajo como el CIMAV y la UACH.

Con todo, el índice de colaboración internacional de los investigadores del estado no parece distanciarse mucho del alcanzado en otras latitudes, si lo comparamos, por ejemplo, con un estudio realizado por Sancho et al (2006), sobre la producción científica de América Latina, en el que se obtuvo un porcentaje del 35.80 .

$\mathrm{El}$ incremento notable de proyectos de colaboración internacional es un fenómeno que ha sido estudiado por autores como Wagner (2005), quien concuerda en que una de las principales razones para este incremento es la búsqueda de los autores de una mayor visibilidad en el contexto mundial. Un punto en el que parece haber controversia es si la colaboración internacional está influida por las áreas del conocimiento abordadas, pero en una primera aproximación parece que existe una mayor colaboración en ciencias básicas y una menor en el área de las ciencias sociales.

La Tabla VII muestra la ubicación geográfica de los investigadores con los que se realizaron los 384 documentos que registraron colaboración internacional. Como es posible advertir, más de la mitad de estas colaboraciones se dieron con colegas adscritos a instituciones de los EUA, siendo España el segundo país con prácticamente una quinta parte de las colaboraciones realizadas con el primer país. Llama la atención que el tercer país con el que más se colabora es Cuba, en tanto que los EUA mantienen bloqueos económicos y de otro tipo hacia ese país, y además porque Francia quedó desplazada a un cuarto lugar. 
Tabla VII. Países con los que Chihuahua ha realizado colaboración internacional, 1999-2008.

\begin{tabular}{|l|c|c|}
\hline \multicolumn{1}{|c|}{ Países } & Frecuencias & Porcentaje \\
\hline EUA & 200 & 52,08 \\
\hline España & 43 & 11,2 \\
\hline Cuba & 31 & 8,07 \\
\hline Francia & 26 & 6,77 \\
\hline Canadá & 23 & 5,99 \\
\hline Argentina & 14 & 3,65 \\
\hline Rusia & 13 & 3,39 \\
\hline Brasil & 12 & 3,13 \\
\hline Inglaterra & 12 & 3,13 \\
\hline Alemania & 6 & 1,56 \\
\hline Colombia & 6 & 1,56 \\
\hline Austria & 5 & 1,3 \\
\hline Perú & 5 & 1,3 \\
\hline Suecia & 5 & 1,3 \\
\hline Venezuela & 5 & 1,3 \\
\hline Ecuador & 4 & 1,04 \\
\hline Israel & 4 & 1,04 \\
\hline Japón & 4 & 1,04 \\
\hline Chile & 3 & 0,78 \\
\hline China & 354,75 \\
\hline Otros & & 0,78 \\
\hline Sumatoria & 32 & 5,73 \\
\hline Total de documentos & 360 \\
\hline
\end{tabular}

Una de las hipótesis que se planteaban al principio de la investigación era que la ubicación geográfica del estado de Chihuahua, en la frontera norte de México y en colindancia con los EUA, podría reflejarse en una incidencia de colaboración con ese país que superaría la que en promedio se realiza en México. $\mathrm{Al}$ respecto, puede decirse que esto se cumple, si se comparan los datos obtenidos con los presentados por CONACyT (2007), donde se observó, que de un total de 24,258 artículos generados en colaboración con otros países, durante el periodo 2002-2006, el 28.4 por ciento se realizó con los EUA; el 8.6 por ciento con España y el 6.6 por ciento con Francia. Sin embargo, cabe reconocer que este mayor índice de colaboración con investigadores norteamericanos no necesariamente puede derivarse de una proximidad geográfica, pues actualmente existen herramientas de comunicación que eliminan distancias; 
se reconoce también el atractivo que significan los altos indicadores de investigación y de difusión científica que se originan en los EUA.

Otro dato muy relacionado es el índice de colaboración institucional, con el cual se determina cuántas instituciones participaron en promedio para que fuera posible obtener cada uno de los documentos producidos. Este indicador, al igual que todos los que tienen que ver con colaboración, está registrando una tendencia a la alza. Como es posible constatar en la Tabla VIII, el conjunto de instituciones objeto de este estudio responden a esta tendencia, pues mientras en 1999 el índice de colaboración era de 2.18 instituciones por trabajo, para el 2008 alcanzaba ya un 2.55.

Tabla VIII: \% de colaboración institucional del estado de Chihuahua, 1999-2008

\begin{tabular}{|l|c|c|c|c|c|c|c|c|c|c|}
\hline \multicolumn{1}{|c|}{ Años } & $\mathbf{1 9 9 9}$ & $\mathbf{2 0 0 0}$ & $\mathbf{2 0 0 1}$ & $\mathbf{2 0 0 2}$ & $\mathbf{2 0 0 3}$ & $\mathbf{2 0 0 4}$ & $\mathbf{2 0 0 5}$ & $\mathbf{2 0 0 6}$ & $\mathbf{2 0 0 7}$ & $\mathbf{2 0 0 8}$ \\
\hline Número de documentos & 28 & 56 & 77 & 60 & 80 & 104 & 130 & 145 & 165 & 128 \\
\hline Número de instituciones & 61 & 126 & 168 & 134 & 200 & 252 & 310 & 346 & 374 & 327 \\
\hline $\begin{array}{l}\text { Grado de colaboración } \\
\text { institucional }\end{array}$ & 2.18 & 2.25 & 2.18 & 2.23 & 2.5 & 2.42 & 2.38 & 2.39 & 2.27 & 2.55 \\
\hline
\end{tabular}

La cifra de 2.55 instituciones por documento puede considerarse como normal, pues en estudios como el ya referido de Sancho et al (2006), relacionado con las ciencias de la salud, se reportaron en promedio 2.4 instituciones participantes por documento.

\subsection{Indice de coautoría}

Otro fenómeno que se está presentando en forma creciente es el incremento de autores por cada documento producido. Según es posible constatar en la Tabla IX la producción científica del estado de Chihuahua no se escapa de esta tendencia: mientras que el índice de coautoría en 1999 era de 3.46 autores por documento, la cifra había llegado en el 2008 a 5.32. La media para los 10 años representa un índice de coautoría de 4.66 autores por documento.

Tabla IX: Índice de coautoría del estado de Chihuahua, 1999-2008

\begin{tabular}{|l|c|c|c|c|c|c|c|c|c|c|}
\hline \multicolumn{1}{|c|}{ Años } & $\mathbf{1 9 9 9}$ & $\mathbf{2 0 0 0}$ & $\mathbf{2 0 0 1}$ & $\mathbf{2 0 0 2}$ & $\mathbf{2 0 0 3}$ & $\mathbf{2 0 0 4}$ & $\mathbf{2 0 0 5}$ & $\mathbf{2 0 0 6}$ & $\mathbf{2 0 0 7}$ & $\mathbf{2 0 0 8}$ \\
\hline Número de documentos & 28 & 56 & $\mathbf{7 7}$ & 60 & 80 & 104 & 130 & $\mathbf{1 4 5}$ & 165 & 128 \\
\hline Número de autores & 97 & 278 & 323 & 262 & $\mathbf{4 0 4}$ & $\mathbf{4 8 4}$ & 623 & $\mathbf{7 1 6}$ & $\mathbf{7 9 6}$ & 681 \\
\hline Índice de coautoría & 3.46 & 4.96 & 4.19 & 4.37 & 5.05 & 4.65 & 4.79 & 4.94 & 4.82 & 5.32 \\
\hline
\end{tabular}


Nuevamente, la cifra no parece ser demasiado alta si se compara por ejemplo con el dato obtenido por Ortiz, Suárez y Sanz (2002) al analizar la producción de científicos de la salud en Puerto Rico en el periodo de 19901998, en que encontraron una media de 5.25 autores por trabajo, aunque cabe aclarar que el índice de coautoría parece variar de una disciplina a otra, siendo las ciencias de la salud las que presentan uno de los índices más altos.

\subsection{Países de publicación}

Otro dato que tiene que ver con la proyección internacional de los investigadores y que afecta la visibilidad de su producción se refiere a la ubicación geográfica de las revistas que se han seleccionado para publicar, información que se presenta en la Tabla X. De nuevo los Estados Unidos aparecen en primer lugar como país sede de la institución editora, con 390 de los documentos publicados, correspondientes a un 40.08 por ciento, seguidos de Inglaterra, con 166 documentos y un 17.06 por ciento. Esto es coherente con el hecho de que la mayor parte de la producción científica internacional se publica en inglés.

Tabla X: Países donde se editan las revistas

\begin{tabular}{|l|c|c|}
\hline \multicolumn{1}{|c|}{ Países } & Núm. De doctos. & Porcentaje \\
\hline EUA & 390 & 40,08 \\
\hline Inglaterra & 166 & 17,06 \\
\hline Holanda & 136 & 13,98 \\
\hline Suiza & 104 & 10,69 \\
\hline México & 67 & 6,89 \\
\hline Alemania & 22 & 2,26 \\
\hline España & 20 & 2,06 \\
\hline Canadá & 12 & 1,23 \\
\hline Venezuela & 8 & 0,82 \\
\hline Italia & 6 & 0,62 \\
\hline Bélgica & 5 & 0,51 \\
\hline Cuba & 3 & 0,31 \\
\hline Grecia & 3 & 0,31 \\
\hline Nueva Zelanda & 3 & 0,31 \\
\hline Rusia & 3 & 0,31 \\
\hline Otros & 25 & 2,57 \\
\hline Totales & 973 & 100 \\
\hline
\end{tabular}


Llama la atención el alto porcentaje de artículos publicados en revistas que aparecen como publicadas en Holanda y Suiza, pero en realidad se trata de revistas editadas por consorcios internacionales como Elsevier, que en ocasiones registran sus domicilios en esos países.

Los títulos de las revistas más utilizadas por los miembros del colectivo estudiado para publicar sus trabajos aparece en la Tabla XI, en la que se agrega también el dato del país donde la revista se publica.

Tabla XI: Títulos de revistas donde más publican los investigadores del estado de Chihuahua, 1999-2008

\begin{tabular}{|l|c|c|c|c|}
\hline \multicolumn{1}{|c|}{ Título de revista } & Frec. & $\%$ & $\%$ Acum. & País de publicación \\
\hline Journal of Molecular Structure-Theochem & 26 & 4,41 & 4,41 & Holanda \\
\hline Salud Pública de México & 13 & 2,2 & 6,61 & México \\
\hline Catalysis Letters & 10 & 1,69 & 8,31 & USA \\
\hline Applied Catalysis a-General & 10 & 1,69 & 10 & Holanda \\
\hline Materials Science and Engineering a- & 9 & 1,53 & 11,53 & Suiza \\
\hline Boletin de la Sociedad Española de Cerámica y Vidrio & 9 & 1,53 & 13,05 & España \\
\hline Agrociencia & 9 & 1,53 & 14,58 & México \\
\hline Interciencia & 8 & 1,36 & 15,93 & Venezuela \\
\hline Thin Solid Films & 7 & 1,19 & 17,12 & Suiza \\
\hline Mycotaxon & 7 & 1,19 & 18,31 & USA \\
\hline Revista Mexicana de Física & 6 & 1,02 & 19,32 & México \\
\hline Materials Letters & 6 & 1,02 & 20,34 & Holanda \\
\hline Otras con menos de 1 por ciento & 470 & & 79,66 & \\
\hline Totales & 590 & & 100 & \\
\hline
\end{tabular}

\subsection{Dispersión}

Con respecto a éste indicador, cabe mencionar que en este caso el análisis se hizo exclusivamente sobre los documentos publicados en formato de artículo.

En la Tabla XII se puede observar que el 63.39 por ciento de la producción se publicó en 106 títulos. El restante 36.61 por ciento de los documentos apareció en 216 revistas. Esto indica una dispersión menor a la reportada por Alonso, Pulgarín y Gil (2006) en el análisis bibliométrico de la producción científica de la Universidad Politécnica de Valencia en 1973-2001, en donde el 48.93 por ciento de las revistas publicó un artículo. Por su parte, Delgado y Russell (1992) muestran que en la UNAM el 50 por ciento de las revistas de su estudio sólo publicaron un artículo. 
Tabla XII: Dispersión de la producción científica del estado de Chihuahua, 1999-2008

\begin{tabular}{|c|c|c|c|c|c|c|}
\hline Títulos & Núm. Art. & Tot. Art. & $\begin{array}{c}\text { Acum. de } \\
\text { revistas }\end{array}$ & $\begin{array}{c}\text { Acum. de } \\
\text { artículos }\end{array}$ & Por ciento & $\%$ Acum. \\
\hline 1 & 26 & 26 & 1 & 26 & 4,41 & 4,41 \\
\hline 1 & 13 & 13 & 2 & 39 & 2,2 & 6,61 \\
\hline 2 & 10 & 20 & 4 & 59 & 3,39 & 10 \\
\hline 3 & 9 & 27 & 7 & 86 & 4,58 & 14,58 \\
\hline 1 & 8 & 8 & 8 & 94 & 1,36 & 15,93 \\
\hline 2 & 7 & 14 & 10 & 108 & 2,37 & 18,31 \\
\hline 2 & 6 & 12 & 12 & 120 & 2,03 & 20,34 \\
\hline 8 & 5 & 40 & 20 & 160 & 6,78 & 27,12 \\
\hline 12 & 4 & 48 & 32 & 208 & 8,14 & 35,25 \\
\hline 18 & 3 & 54 & 50 & 262 & 9,15 & 44,41 \\
\hline 56 & 2 & 112 & 106 & 374 & 18,98 & 63,39 \\
\hline 216 & 1 & 216 & 322 & 590 & 36,61 & 100 \\
\hline
\end{tabular}

Como se puede advertir, en 322 títulos se dieron a conocer los 590 artículos y en 67 de ellos se publicó el 50.17 por ciento. La revista más utilizada para difundir sus trabajos aporta el 4.41 por ciento. Se aprecia que hay 10 títulos de revistas iberoamericanas que publicaron un 10.36 por ciento de los trabajos.

\subsection{Impacto y documentos citados}

A partir de los datos de la Tabla XIII se puede describir el impacto de la producción científica del estado de Chihuahua, con base en el análisis de 3 años no consecutivos, los cuales representan el 33.47 por ciento de la producción. Se calculó una media que indica que el 50.61 por ciento corresponde a los cuartiles 1 y 2 . Por su parte, los cuartiles 3 y 4 aportaron el 49.39 por ciento. Lo que quiere decir, que solamente la mitad de la producción, goza de un buen impacto y visibilidad internacional.

Tabla XIII. Impacto de la producción científica del estado de Chihuahua, 1999-2008

\begin{tabular}{|c|c|c|c|c|c|c|}
\hline $\begin{array}{c}\text { Años } \\
\text { revisados }\end{array}$ & $\begin{array}{c}\text { Total de } \\
\text { títulos }\end{array}$ & $\begin{array}{c}\text { Títulos sin factor } \\
\text { de impacto }\end{array}$ & $\begin{array}{c}\text { Cuartil } \\
\mathbf{1}\end{array}$ & $\begin{array}{c}\text { Cuartil } \\
\mathbf{2}\end{array}$ & $\begin{array}{c}\text { Cuartil } \\
\mathbf{3}\end{array}$ & $\begin{array}{c}\text { Cuartil } \\
\mathbf{4}\end{array}$ \\
\hline 1999 & 16 & 1 & 5 & 4 & 4 & 2 \\
\hline 2003 & 57 & 2 & 13 & 16 & 17 & 9 \\
\hline 2008 & 91 & 3 & 15 & 30 & 21 & 22 \\
\hline \multirow{2}{*}{ Totales } & $\begin{array}{c}164 \\
(33,47 \%)\end{array}$ & $\begin{array}{c}6 \\
(3,66 \%)\end{array}$ & $\begin{array}{c}33 \\
(20,12 \%)\end{array}$ & $\begin{array}{c}50 \\
(30,49 \%)\end{array}$ & $\begin{array}{c}42 \\
(25,12 \%)\end{array}$ & $\begin{array}{c}33 \\
(20,12 \%)\end{array}$ \\
\hline
\end{tabular}


Los resultados muestran que los autores Paraguay, F.; Estrada, W.; Acosta, D. R.; Andrade, E. y Miki-Yoshida, M., con el artículo: "Growth, Structure and Optical Characterization of High Quality Zno Thin Films Obtained by Spray Pyrolysis", han sido citados en 141 ocasiones, lo que equivale al 5.16 por ciento.

Por su parte la tabla la Tabla XIV muestra que la producción científica de artículos tiene 2,733 citas, en donde 202 documentos aparecen sin ninguna cita y 101 con una sola, lo que quiere decir que entre ambos obtienen una escasa o nula citación y representan el 51.35 por ciento de la producción analizada.

Tabla XIV. Citas de los artículos de revistas

\begin{tabular}{|c|c|c|}
\hline Documentos & $\begin{array}{l}\text { Núm. De citas } \\
\text { recibidas }\end{array}$ & Citas acumuladas \\
\hline 1 & 141 & 141 \\
\hline 1 & 106 & 106 \\
\hline 1 & 77 & 77 \\
\hline 2 & 47 & 94 \\
\hline 1 & 36 & 36 \\
\hline 1 & 35 & 35 \\
\hline 2 & 33 & 66 \\
\hline 2 & 29 & 58 \\
\hline 2 & 27 & 54 \\
\hline 2 & 25 & 50 \\
\hline 2 & 23 & 46 \\
\hline 4 & 22 & 88 \\
\hline 1 & 21 & 21 \\
\hline 4 & 20 & 80 \\
\hline 1 & 19 & 19 \\
\hline 4 & 18 & 72 \\
\hline 4 & 17 & 68 \\
\hline 3 & 16 & 48 \\
\hline 6 & 15 & 90 \\
\hline 10 & 14 & 140 \\
\hline 5 & 13 & 65 \\
\hline 7 & 12 & 84 \\
\hline 7 & 11 & 77 \\
\hline 9 & 10 & 90 \\
\hline 8 & 9 & 72 \\
\hline 17 & 8 & 136 \\
\hline
\end{tabular}




\begin{tabular}{|c|c|c|}
\hline 18 & 7 & 126 \\
\hline 24 & 6 & 144 \\
\hline 26 & 5 & 130 \\
\hline 29 & 4 & 116 \\
\hline 37 & 3 & 111 \\
\hline 46 & 2 & 92 \\
\hline 101 & 1 & 101 \\
\hline 202 & 0 & 0 \\
\hline 590 & 832 & 2733 \\
\hline
\end{tabular}

\section{Conclusiones}

1. El estado de Chihuahua no tiene un nivel de desarrollo en investigación científica acorde con su desarrollo económico, cabe esperar que las políticas implementadas recientemente logren resultados en el mediano plazo.

2. La producción científica del estado se polariza en cuatro instituciones, el Centro de Investigación en Materiales Avanzados, la Universidad Autónoma de Chihuahua, la Universidad Autónoma de Ciudad Juárez y el Instituto Tecnológico de Chihuahua, que en conjunto tienen participación en el 92.08 por ciento de la producción.

3. Se comprueba un cierto perfil en cuanto a la temática que más interesa a los investigadores del estado, aunque este perfil se encuentra sesgado por la producción del CIMAV. Por otra parte, aunque sería motivo de un estudio más detallado, al parecer la producción científica del estado no constituye todavía un soporte sólido para las actividades económicas a las que el estado pretende darles prioridad, como son los esfuerzos recientes por constituirse en un polo industrial especializado en aeronáutica.

4. La tipología encontrada en el estudio presenta una diversidad de documentos, aunque los porcentajes de algunos de ellos son escasamente representativos. En los resultados del estudio se evidenció la influencia del uso de las bases de datos utilizadas, como la inclusión de las que registran proceedings, que seguramente influyeron para que el 25.59 por ciento fuera para la tipología Actas de congresos y el 10.48 por ciento para los Resúmenes de comunicaciones.

5. Se demuestra en el estudio una tendencia de los investigadores locales, con respecto al promedio nacional, para aprovechar la cercanía y la relación con instituciones de los EUA y para publicar la mayor parte 
de su producción en idioma inglés.

6. Existe una importante área de oportunidad por continuar incrementando la colaboración entre las instituciones que realizan investigación dentro del estado, sin descuidar la colaboración con otras instituciones del país o internacionales.

7. Las revistas donde más publica el colectivo mostró que en el 32.9 por ciento de los títulos se publicó el 63.39 por ciento de los documentos; de igual manera, se pudo observar que pocos artículos concentran la mayor cantidad de citaciones.

8. En el análisis del impacto, las revistas iberoamericanas contribuyeron con 11 títulos, la mayoría de ellos con un factor de impacto bajo, ubicando al 90 por ciento de éstos en el cuarto cuartil.

9. Es necesario identificar o desarrollar un sistema que permita darle un mejor seguimiento a la producción de conocimiento en el área de las Ciencias sociales y las Humanidades en el contexto nacional y estatal.

\section{BiBLIOGRAFÍA}

Adam, D. (2002), “The Counting House”, en Nature 415, núm. 14: pp. 726-29.

Alonso, AA., Pulgarín, A. y Gil, LI (2006), “Análisis bibliométrico de la producción científica de la Universidad Politécnica de Valencia 1973-2001", en Revista Española de Documentación Científica 29, núm. 3: pp. 345-63.

Braun, T., Glänzel, SW., y Schubert, A (1985), A Scientometric Indicators. A 32- Country Comparative Evaluation of Publishing Performance and Citation Impact, Singapure: World Scientific Publisher.

Cami, J. et al. (2003), "Producción científica española en biomedicina y ciencias de la salud. Mapa bibliométrico de la investigación realizada en España durante el período 1994-2000”, en Quark núm. 30: pp. 17-26.

Consejo Nacional de Ciencia y Tecnología (2007), Informe general del estado de la Ciencia y la Tecnología, México: El Consejo, 380 p. (2008), Estado del arte de los sistemas estatales de ciencia y tecnología: Chibuabua 2007, México: CONACYT, 34 p. (2008b), Sistema de Centros de Investigación CONACYT, México: El Consejo, 72 p.

(2009), "Índice de Revistas Mexicanas de Investigación Científica y Tecnológica”, disponible en: http://www.conacyt. $\mathrm{mx} /$ Indice/Index_Indice.html, recuperado el 29 de julio de 2009.

Cronin, B., y Overfelt, K (1994), "Citation Based Auditing of Academic Performance”, en JASIS 45, núm. 2: pp. 61-72. 
Delgado, H., y Russell JM. (1992), "Impact of Studies Published in the International Literature by Scientists at the National University of Mexico", en Scientometrics 23, núm. 1: pp. 75-90.

Fernández, M MT., Gómez CI, y Sebastián, J. (1998), “La cooperación científica de los países de América Latina a través de indicadores bibliométricos", en Interciencia 23, núm. 6: pp. 328-37.

Fernández, MMT, et al (2004), Indicadores de especialización temática de los países de América Latina (Taller de Indicadores de Ciencia y Tecnología VI: Buenos Aires: 9- 11 septiembre: 2004), disponible en: http://www.ricyt.org/interior/ormalización/Vitaller/ S5_produc/fernandezdoc.pdf, recuperado el 16 de febrero de 2009.

Gálvez, C., y Moya AF. (2006), “The Unification of Institutional Addresses Applying Parametrized Finite-state Graphs (P-FSG)", en Scientometrics 69, núm. 2: pp. 323-45.

González, E., Gómez HJA y Licea de Arenas J. (2008), “La visibilidad internacional de la investigación científica: el caso de la Universidad Nacional Autónoma de México", en BID Textos Universitaris de Biblioteconomia i Documentació, núm. 21.

Lange, LL. (2001), "Citation counts of multi-authored papers -firstname authors and futher authours", en Scientometrics 52, núm. 3: pp. 457-70.

Lascurain, SML. (2001), "Análisis de la actividad científica y del consumo de información de los psicólogos españoles del ámbito universitario durante el período 1986-1995”, Carlos III de Madrid, Tesis doctoral, Universidad Carlos III de Madrid, Facultad de Humanidades Comunicación y Documentación, Departamento de Biblioteconomía y Documentación.

Leta, J., J.C. R. Pereira y H. Chaimovich. 2005, “The life sciences - the relative contribution of the University of São Paulo to the highest impact factor journals and to those with the largest number of articles, 1980 to 1999", en Scientometrics 63, núm. 3: pp. 599-616.

Licea de Arenas, J., et al (2006), "Investigación mexicana significativa en ciencias de la salud 1999-2004. Un análisis bibliométrico”, en Anales de Documentación. Núm. 9: pp. 123-32.

Maltrás, BB. (2003), Los indicadores bibliométricos : fundamentos y aplicación al análisis de la ciencia, Gijón: Trea.

Moreno, CF. (2010), Producción científica de los investigadores de la Universidad de Guadalajara reportada en el ISI Web of Knowledge, durante el periodo 1996-2005: un análisis bibliométrico desde el modelo departamental. nota: Tesis doctoral, Universidad Carlos III de Madrid, Facultad de Humanidades Comunicación y Documentación, Departamento de Biblioteconomía y Documentación.

Olmeda, GC. et al (2008), "Impacto internacional de la investigación y la colaboración científica de las universidades de Cataluña. 2000-2004", en Revista Española de Documentación Científica 31, núm. 4: pp 591-611. 
Ordorika, SI. et al (2009), "Desempeño de universidades mexicanas en la función de investigación: Estudio comparativo", en Cuadernos de Trabajo de la Dirección General de Evaluación Institucional, año 1, núm. 2, DGEI - UNAM, México, D. F.

Ortiz RLA (2002), "Estudio bibliométrico de la producción científica de autores pertenecientes a instituciones puertorriqueñas en el Science Citation Index durante el periodo 1980-1998", Carlos III de Madrid, nota: Tesis doctoral, Universidad Carlos III de Madrid. Facultad de Humanidades Comunicación y Documentación, Departamento de Biblioteconomía y Documentación.

Ortiz, RLA., Suárez, BC., y Sanz, CE (2002), "Enfoque bibliométrico de la producción científica en ciencias de la salud en Puerto Rico, a través de la base de datos Science Citation Index durante el período de 1990 a 1998", en Revista Española de Documentación Cientifica 25, núm. 1: pp. 9-28.

Russell, JM., Ainswort, S. y Narváes BN (2006), “Colaboración científica de la Universidad Nacional Autónoma de México (UNAM) y su política institucional”, en Revista Española de Documentación Cientifica 29, núm. 1: pp. 56-73.

Sancho, R. et al (2006), "Indicadores de colaboración científica intercentros en los países de América Latina”, en Interciencia 31, núm. 4: pp. 284-92.

Taubes, G. (1993), “Measure for Measure in Science”, en Science 260: pp. 884-86.

Wagner, CS. (2005), "Six Case Studies of International Collaboration in Science”, en Scientometrics 62, núm. 1: pp. 3-26. 
ANEXO:

\section{Acrónimos utilizados}

Nota: únicamente se enlistan los que se mencionan más de una vez.

CIAD-CH Centro de Investigación en Alimentación y Desarrollo del Estado de Chihuahua

CIMAV: $\quad$ Centro de Investigación en Materiales Avanzados

CONACYT: Consejo Nacional de Ciencia y Tecnología

EUA: $\quad$ Estados Unidos de América

IMSS-CH: Instituto Mexicano del Seguro Social, delegación Chihuahua

INIFAP-CH: Instituto Nacional de Investigaciones Forestales, Agrícolas y Pecuarias, Campo

Experimental Chihuahua

ISI: $\quad$ Institute for Scientific Information

ITECH: Instituto Tecnológico de Chihuahua

ITECH 2: Instituto Tecnológico de Chihuahua, plantel 2

ITESM-CH: Instituto Tecnológico y de Estudios Superiores de Monterrey, Campus

Chihuahua

SNI: $\quad$ Sistema Nacional de Investigadores

UACH: $\quad$ Universidad Autónoma de Chihuahua

UACJ: $\quad$ Universidad Autónoma de Ciudad Juárez

UNAM: $\quad$ Universidad Nacional Autónoma de México 
